\title{
Solar rotational cycle in lightning activity in Japan during the 18-19th centuries
}

\author{
Hiroko Miyahara ${ }^{1}$, Ryuho Kataoka ${ }^{2,3}$, Takehiko Mikami ${ }^{4}$, Masumi Zaiki $^{5}$, Junpei Hirano ${ }^{4}$, Minoru Yoshimura ${ }^{6}$, \\ Yasuyuki Aono ${ }^{7}$, and Kiyomi Iwahashi ${ }^{8}$ \\ ${ }^{1}$ Humanities and Sciences/Museum Careers, Musashino Art University, 1-736 Ogawa-cho, \\ Kodaira-city, Tokyo 187-8505, Japan \\ ${ }^{2}$ National Institute of Polar Research, 10-3, Midori-cho, Tachikawa-city, Tokyo 190-8518, Japan \\ ${ }^{3}$ Department of Polar Science, School of Multidisciplinary Sciences, Sokendai 10-3 Midori-cho, \\ Tachikawa-city, Tokyo 190-8518, Japan \\ ${ }^{4}$ Faculty of Liberal Arts, Teikyo University, 359 Ohtsuka, Hachioji city, Tokyo 192-0395, Japan \\ ${ }^{5}$ Faculty of Economics, Seikei University, 3-3-1 Kichijoji-Kitamachi, Musashino-city, Tokyo 180-8633, Japan \\ ${ }^{6}$ Faculty of Education, University of Yamanashi, 4-4-37 Takeda, Kofu-city, Yamanashi 400-8510, Japan \\ ${ }^{7}$ Graduate School of Life and Environmental Sciences, Osaka Prefecture University, 1-1 Gakuen-cho, \\ Naka-ku, Sakai-city, Osaka 599-8531, Japan \\ ${ }^{8}$ National Institute of Japanese Literature, 10-3, Midori-cho, Tachikawa-city, Tokyo 190-0014, Japan
}

Correspondence: Hiroko Miyahara (miyahara@musabi.ac.jp)

Received: 13 December 2017 - Revised: 12 March 2018 - Accepted: 21 March 2018 - Published: 18 April 2018

\begin{abstract}
Thunderstorm and cloud activities sometimes show a 27-day period, and this has long been studied to uncover a possible important link to solar rotation. Because the 27-day variations in the solar forcing parameters such as solar ultraviolet and galactic cosmic rays become more prominent when the solar activity is high, it is expected that the signal of the 27-day period in meteorological phenomena may wax and wane according to the changes in the solar activity level. In this study, we examine in detail the intensity variations in the signal of the 27-day solar rotational period in thunder and lightning activity from the 18th to the 19th centuries based on 150-year-long records found in old diaries kept in Japan and discuss their relation with the solar activity levels. Such long records enable us to examine the signals of solar rotation at both high and low solar activity levels. We found that the signal of the solar rotational period in the thunder and lightning activity increases as the solar activity increases. In this study, we also discuss the possibility of the impact of the long-term climatological conditions on the signals of the 27-day period in thunder/lightning activities.
\end{abstract}

Keywords. Meteorology and atmospheric dynamics (lightning)

\section{Introduction}

Thunderstorm activity sometimes shows a period of approximately 27 days, which is comparable to the solar rotational period. The 27-day period has been detected in thunder and lightning activity, for example, in the records in England for AD 2000-2005 (Scott et al., 2014) and AD 2000-2007 (Owens et al., 2015) and in Japan for AD 1991-1992 and AD 1999-2001 (Muraki et al., 2004). A relatively longer record of thunder and lightning activity since AD 1989 in Japan has also shown a signal of solar rotational period near the maxima of the solar decadal cycle (Miyahara et al., 2017a). It has also been suggested that cloud activities in tropical areas had a similar periodicity at the solar cycle maxima during the period of AD 1980-2003 (Takahashi et al., 2010; Hong et al., 2011); this may also be related to the solar rotation. The amplitudes of the Schumann resonance, which are excited by lightning activity, also exhibit a solar rotational period near the maxima of solar decadal cycles, e.g., in AD 1990 (Füllekrug and Fraser-Smith, 1996) and during the period of AD 2000-2002 (Sato and Fukunishi, 2005), although the Schumann resonance is also influenced by the change in the ionospheric conditions that are under the impact of solar activity and thus it may not be directly reflecting 
the global lightning activity. It has also been indicated that ionospheric conditions themselves are influenced by lightning (Davis and Johnson, 2005).

The mechanisms for a solar influence on the climate and weather are not certain; however, possible explanations are given based on the forcing of solar radiation (Foukal, 2004, 2006; Hood, 1986; Shindell, 1999; Kodera and Kuroda, 2002; Zhou and Tung, 2013), galactic cosmic rays (Svensmark and Friis-Christensen, 1997; Marsh and Svensmark, 2000; Yamaguchi et al., 2010; Svensmark et al., 2016), solar energetic particles (Jackman et al., 2009; Scott et al., 2014), and from the changes in the atmospheric electric circuit (Tinsley, 1996; Owens et al., 2015). The variations of solar-related parameters associated with solar rotations are quasi-periodic. For example, solar radiative outputs vary in time with a period of 24-31 days due to the migration of sunspots and faculae on the solar surface. Due to the occurrence of coronal mass ejections or the passage of co-rotating interaction regions on Earth, the flux of galactic cosmic rays shows an anomaly of a few days every 26-31 days.

The amplitude of monthly-scale variations in solar-related parameters such as solar ultraviolet (UV) and galactic cosmic rays become larger as the level of magnetic activity and number of sunspots at the solar surface increase according to the decadal-scale cycles of the solar activity. Therefore, it is expected that if the signal of the 27-day period in meteorological phenomena has an origin in such parameters, it will become more prominent as the level of solar activity is enhanced. This origin has not yet been confirmed; however, examinations of the signal of 27-day cycles in meteorological phenomena could identify the extent of the solar forcing and, more importantly, clarify the mechanisms and propagation of the solar influence. For example, in a previous study, it was shown that the signal of the 27-day period indicated a pattern of northeastward migration in Japan, suggesting that the signal may have propagated from lower latitudes (Miyahara et al., 2017a).

The above examples of the detection of a 27-day period in thunder and lightning activity are, however, limited to a short period of less than a few decades in the modern era. To test the possibility of detecting monthly-scale periodicity in thunder and lightning records from the premodern era, in a recent study, we analyzed a record retrieved from old diaries kept in Kyoto (Miyahara et al., 2017b). Despite the limited number of events due to the likely lower sensitivity of the record keepers to thunder and lightning events compared to that of the modern era with systematic audio (thunder) and optical (lightning) observations, we successfully detected a 27-day period in the thunder and lightning activity during the period of AD 1716-1767. The record exhibited a possible contrast in the signals between the periods of high and low solar activity levels.

In this study, we examine 150 years of multiple thunderstorm records using old diaries in which weather information was regularly recorded. We examine two diaries in Tokyo written in the 18th and 19th centuries and in parts of the 17th century and one diary in Hirosaki in northern Japan, which is available from the late 17 th to mid-19th centuries. The period from the 18th to the 19th centuries includes the times of both low and high activity levels in the centennial-scale variability, and the lengths of the records are sufficient to test the significance of the signal of the 27-day period for each solar activity level. In addition, the examination of diaries from multiple locations enables us to discuss the possible change in the spatial coverage of the influence of solar rotation.

\section{Analysis methods}

To analyze the periodicity of the thunder and lightning activity in the past, we used weather records in two series of diaries written in Tokyo. The first one is the series kept by the Ishikawa family (since AD 1720) currently located in Hachioji City in the western part of Tokyo Prefecture. The diaries are preserved in the Hachioji City Historical Museum and are available for inspection (in-library use only). Books on the contents of the diaries entitled "Ishikawa Nikki (vol. 1-15)" have been published and are also available at the museum. A database of the weather records extracted from the diaries was constructed by Yoshimura in 2006 and is available at https://tk2-202-10627.vs.sakura.ne.jp/ (last access: 10 April 2018). The other diary is the series kept by the Hirosaki Domain currently located in the Aomori Prefecture, the northernmost prefecture of Honshu, the main island of Japan. In the Edo period, Japanese territorial lords were subjected to Sankin-kōtai and were required to spend several months in Edo (currently located in Tokyo) in alternate shifts. The lord of the Hirosaki Domain participated in Sankin-kōtai starting in AD 1668. The lord came to Edo every other year; however, his legal wife and his heir had to remain in Edo. Several officials also stayed in Edo in alternate shifts. In Edo, the record keepers of the Hirosaki Domain kept diaries of the weather, the content of the lord's meals, fire disasters, and floods and so on (Yoshimi Fukuma, 2017, personal communication). They also kept diaries in Hirosaki, which included broader content such as earthquakes, snow slides, damage by wolves, and river freezing events (Fukuma, 2010, 2014). These diaries are currently preserved in the Hirosaki City Library and are available for inspection as well (in-library use only). The weather records in Edo documented by the Hirosaki Domain cover parts of the period of AD 1668-1867 and the detailed descriptions have been extracted back to AD 1710 (Yoshimi Fukuma, 2017, personal communication). The record of weather in Hirosaki kept by the Hirosaki Domain covers parts of the period of AD 16611868. Continuous recordings started in AD 1665; therefore, the records for AD 1665-1868 were used in this study.

We searched for thunder and lightning events in the above diaries and made a list of the dates of their occurrences. In the diaries kept by the Ishikawa family, the events 
were recorded with the terms such as "Kaminari (thunder and lightning)", "Raiu" (thunderstorm), "Raimei (thunder)", "Nari-mono (thunder)", and "Hikari-mono (lightning)". In the diaries by the Hirosaki Domain, the events were recorded with terms such as "Kaminari", "Raiu", "Den/Denkou (lightning)", and "Inabikari (lightning)". We made a list of dates by combining all such events.

We counted both daytime and nighttime events. In the diaries kept by the Hirosaki Domain, most of the thunder and lightning records were accompanied by information concerning the approximate time of occurrence (the temporal hour system was used in the Edo period). On the other hand, the thunder and lightning events recorded by the Ishikawa family were in many cases without information on the time of occurrence. The diaries kept by the Ishikawa family were mainly aimed at recording the summary of the weather and the activities of the day (farm work etc.), and the descriptions of weather are much simpler compared to those by the Hirosaki Domain. In most of the cases, the weather was described by a single phrase such as "fine weather" or "rainy weather".

Based on the extracted data, we conducted periodicity analyses. As has been shown by Miyahara et al. (2017a), the possible signal of the solar rotational period is prominent only in the summertime (approximately May-September) and becomes weak in winter when the climate is strongly influenced by the cold Siberian air mass. Therefore, we extracted the data from May to September in this study. Figure 1 shows the time series of the number of thunder/lightning days for May to September as extracted from the diaries in Hachioji, Edo, and Hirosaki. The gray highlights indicate the periods where the recordings had been paused or the years that have interruptions for $\geq 30$ days.

The three time series show interannual to multidecadal variations. The data in Edo and Hirosaki recorded by the Hirosaki Domain do not show any significant long-term trend; however, the data in Hachioji recorded by the Ishikawa family show a long-term decreasing trend in the total number of thunder/lightning days. Because the distance between Edo and Hachioji is only approximately $40 \mathrm{~km}$, the difference in the trends between the two sites is likely due to changes in the diary keepers, who have various sensitivities and/or depths of interest to thunder and lightning events relative to each other. This would alter the criteria for notable weather events to be recorded in their diaries; therefore, the number of days with specific weather might show long-term trends that can be attributed to changes in the diary keepers. In fact, the thunder and lightning events in the record of Hachioji become sparse after $\mathrm{AD} 1850$, although the diaries continue until the beginning of the 20th century. We therefore conduct the analyses only before AD 1868, the end of the Edo period. Note that the noticeable continual absence of records occurs only in $\mathrm{AD} 1749,1752$, and 1848 (about 40 days for each) during the period analyzed. As in our previous study (Miyahara et al., 2017b), we aim to detect monthly-scale cyclic behav- (a) Hachioji
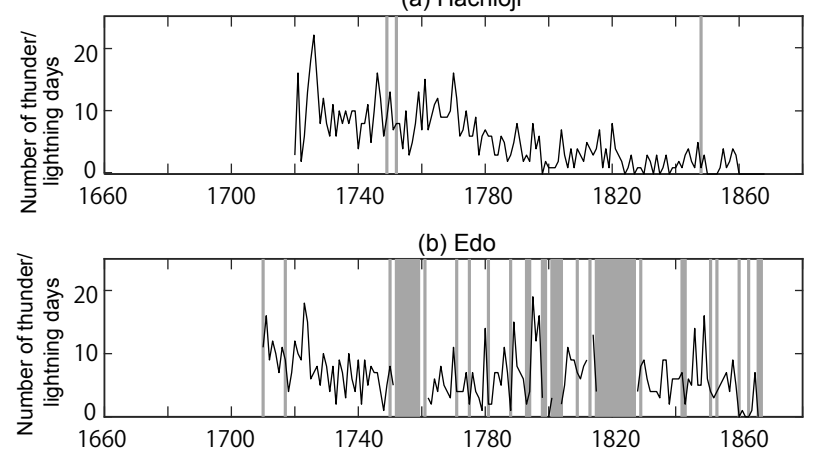

(c) Hirosaki

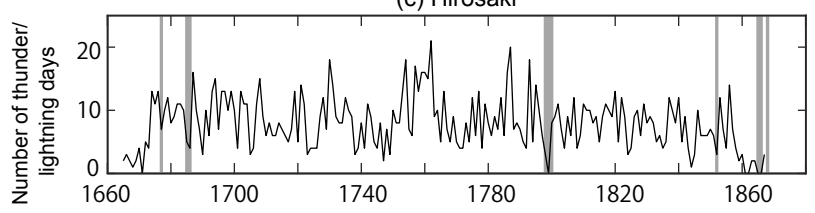

(d)

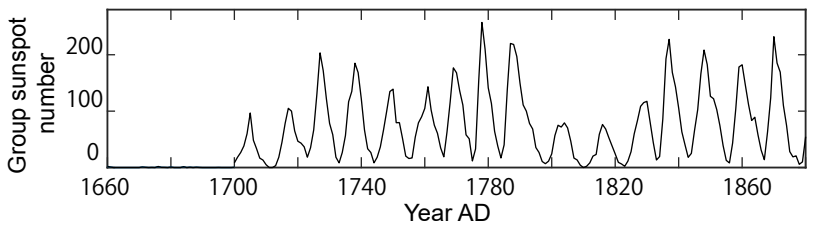

Figure 1. Time series of the number of thunder/lightning days from May to September extracted from old diaries in (a) Hachioji, (b) Edo, and (c) Hirosaki and the time series of (d) the group sunspot numbers (GSNs) for the periods of AD 1650-1700 (Hoyt and Schatten, 1998) and AD 1700-1900 (Clette and Lefevre, 2016). The gray highlights in (a-c) indicate the years when there were interruptions of the recordings for $\geq 30$ days.

ior of thunder and lightning occurrences; therefore, we will not further discuss the long-term trends.

Variations in the solar radiation are associated with magnetic activity of sunspots on the solar surface. The variations of galactic cosmic rays incident to the Earth are caused by the changes in the heliospheric magnetic field, including the ones associated with coronal mass ejections originating from sunspots. Because the lifetimes of sunspots are a few months at most, the phase of the 27-day period in the above parameters is variable in time. In such a case, typical methods of frequency analysis such as the fast Fourier transform (FFT) or the Lomb-Scargle periodogram are not suitable since frequent phase shifts dilute the signals. Therefore, as in previous studies (Miyahara et al., 2017a, b), we conducted a histogram analysis of the thunder and lightning record. To reveal the typical waiting time of the thunder/lightning days, we obtained a histogram by counting the number of days between every combination of two thunder/lightning days. For example, if thunder/lightning events were recorded on $1 \mathrm{Au}-$ gust, 28 August, and 24 September, then counts would be increased by 2 for 27 days and by 1 for 54 days. Note that 


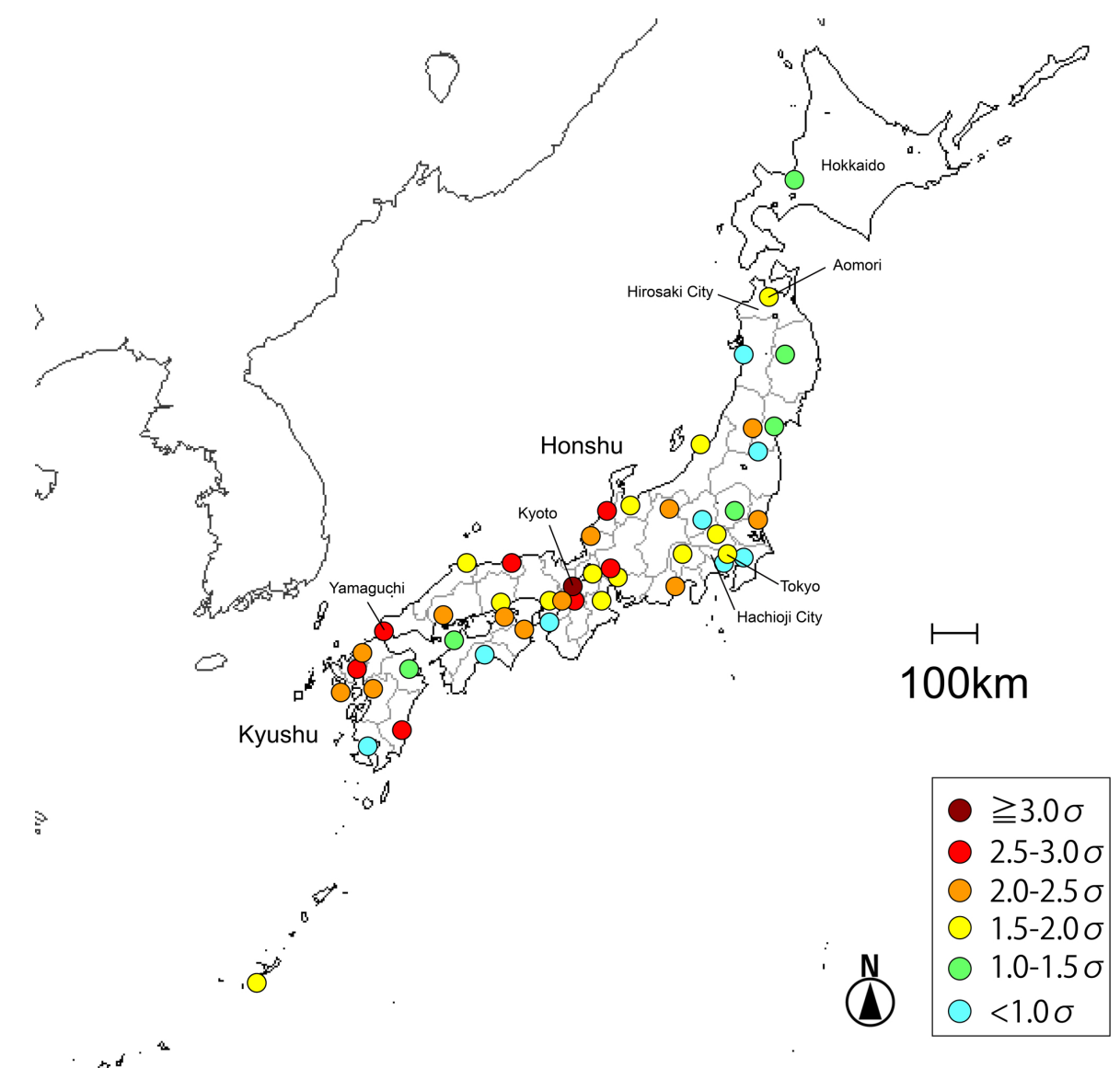

Figure 2. Significance level of the solar rotational period detected in the summertime thunder/lightning data for the periods of solar cycle maxima during the period of AD 1989-2014.

the counts would also be increased by 2 for 27 days in the case where the thunder/lightning events were recorded on 1 , 2, 28, and 29 August. In that case, histogram counts would also be increased by 2 for 1 day, by 1 for 26 days, and by 1 for 28 days. In this method, the above two cases cannot be distinguished. If the thunder/lightning days are recurrent with a 27-day period similar to the above two cases, we would see a peak in the histogram of the waiting time (days). The significance levels of the peaks were calculated based on the standard deviation (SD or $\sigma$ ) of the count-rate anomaly from the best-fit linear slope for a period of $\leqq 100$ days. We excluded the periods for which data gaps are 30 days (indicated by the gray highlights in Fig. 1) from the analyses, since they could have a different background level. Note that the data gaps are continuous in most cases. The data gaps were rare other than the periods indicated.

We applied the histogram analysis for three different time periods: high solar activity (group sunspot numbers, GSNs, are greater than or equal to 150 ), middle solar activity (75 to $150 \mathrm{GSNs}$ ), and low solar activity (less than $75 \mathrm{GSNs}$ ). Figure $1 \mathrm{~d}$ shows the time series of the GSNs (Clette and Lefevre, 2016) for the 18th to 19th centuries. Prior to AD 1700, sunspots were sparse due to the long-term weakening of the solar activity, called the Maunder Minimum (Eddy, 1976). Using the record of Hirosaki, we were able to obtain a histogram during the time interval of AD 1665-1699 when the sunspot activity was extremely weak.

Before examining the thunder and lightning records in the diaries, we investigated the spatial distribution of the signal of the solar rotational period in the thunder/lightning data during the modern era. The dates of thunder/lightning events are distributed by the Japan Meteorology Agency (http: //www.data.jma.go.jp/obd/stats/etrn/, last access: 10 April 2018). Continual daily data are available since AD 1989 for all 47 prefectures of Japan. We extracted data for the periods of solar activity maxima during the past three solar cycles (AD 1989-1991, 1999-2002, and 2011-2014) for each prefecture. The data from May to September were analyzed to construct the histograms. 

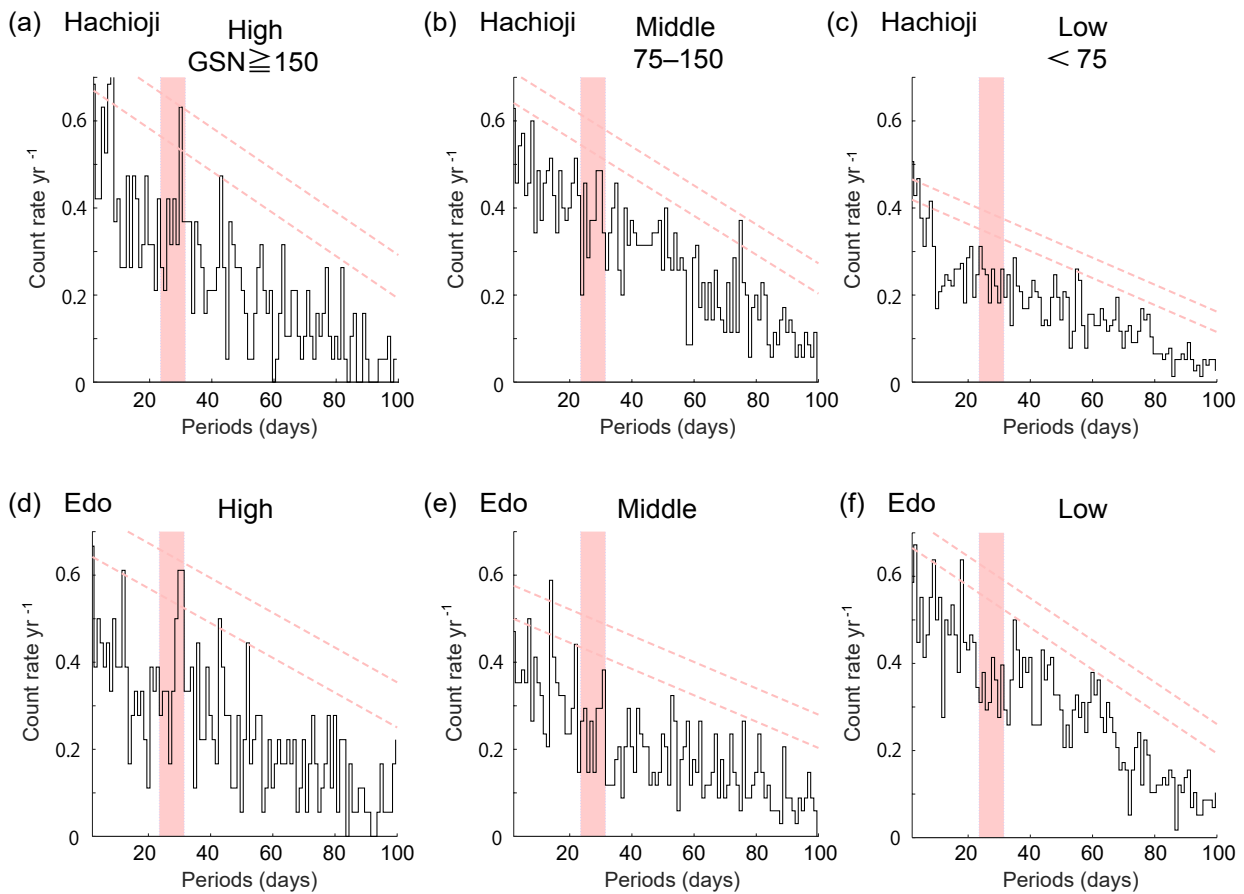

Figure 3. Periodicity of the thunder/lightning events from May to September in (a-c) Hachioji and in (d-f) Edo in the 18th to 19th centuries. Histograms were obtained for the periods when the GSNs were (a, d) greater than or equal to 150, (b, e) from 75 to 150 , and (c, f) less than 75. The red dashed lines denote 2.0 and 3.0 SDs. The red shaded bars indicate the $24-31$ day period.

\section{Results and discussion}

Figure 2 shows the spatial distribution of the significance level of the solar rotational period in the summertime thunder/lightning activity at the solar cycle maxima since AD 1989. The significance level of the signal for each prefecture is shown by a colored circle. The peaks near 24 31 days may be related to solar variations. Several prefectures show a signal of more than 1.5 SDs. Even though the previous study had shown that wide-area thunder/lightning activity covering more than 15 prefectures has a highly significant signal of approximately 4.2 SDs (Miyahara et al., 2017a), the local thunder/lightning activity also shows significant signals. The areas of relatively high significance of more than 2.0 SDs are located primarily around Kyushu and the western to central part of Honshu. Conversely, the signals are less than 2.0 SDs in most of the prefectures in the northern part of Honshu and in Hokkaido, where rain-front activity is rare. More specifically, the significance levels of the signal in Tokyo and Aomori are approximately 1.9 and 1.7 SDs, respectively. Below, we will analyze the old records in Tokyo and in Hirosaki City in the Aomori Prefecture.

Figure 3 shows the results of a histogram analysis on the thunder/lightning record from AD 1720 to 1884 in Hachioji (Fig. 3a-c) and from AD 1710 to 1866 in Edo (Fig. 3d-f); both are currently located in the Tokyo Prefecture. The data were independently analyzed for high solar activity (Fig. 3a and d), middle solar activity (Fig. 3b and e), and low solar activity (Fig. 3c and f). We found similar characteristics in the records in both Hachioji and Edo and found that the signals of the solar rotational period are significant when the sunspot activity level is high (GSNs $\geq 150$ ). The significance levels of the signals are approximately 3.0 and 2.8 SDs for Hachioji and Edo, respectively. When the GSNs are between 75 and 150, the significance levels of the signals are approximately 1.6 and 1.6 SDs, respectively, which is less significant compared to the periods of high solar activity. The signals become insignificant and comparable to the noise level during the periods when the GSNs are less than 75. It is confirmed that the signal of the solar rotational period in thunder/lightning activity extends back to the 18th century and that its intensity clearly depends on the level of sunspot activity. Therefore, it supports the idea that the 27-day period in thunder/lightning activity originates in the solar rotation and its associated activities.

In addition, we conducted the same histogram analysis for the record in the Hirosaki Domain, currently located in the Aomori Prefecture, the northernmost prefecture of Honshu. Figure 4 shows the results of the histogram analyses for the periods of (a) high solar activity, (b) middle solar activity, and (c) low solar activity since AD 1700. Because the record in Hirosaki covers more than 20 years of sunspot absence prior to $\mathrm{AD} 1700$, we also conducted the analysis for the period of AD 1665-1699 (Fig. 4d). Contrary to the case in 

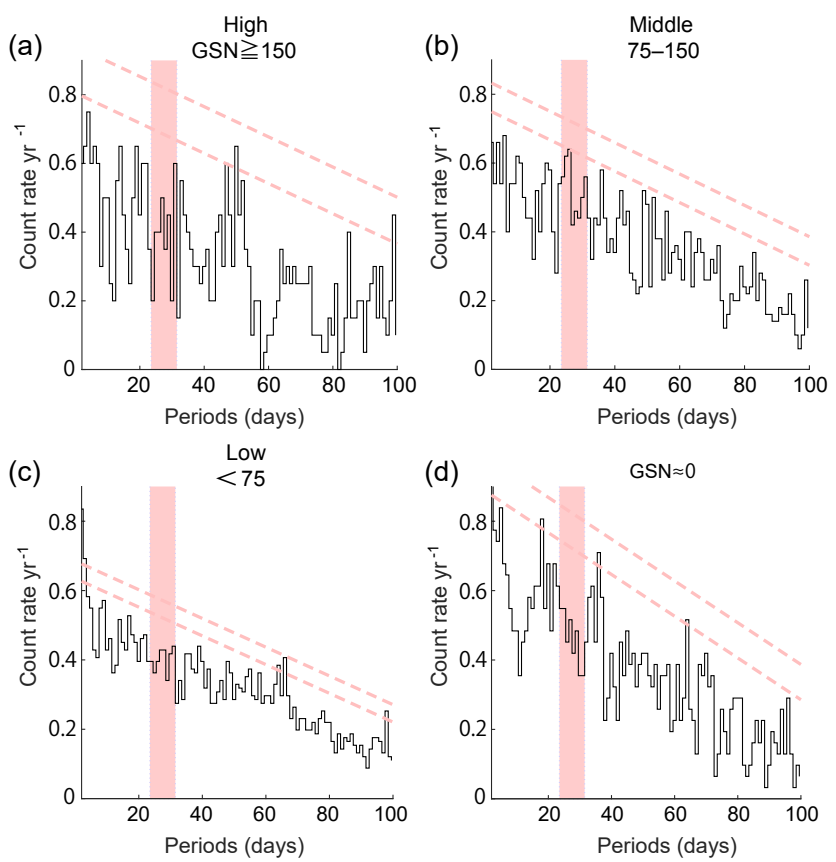

Figure 4. Periodicity of the thunder/lightning events from May to September in Hirosaki for the periods when the GSNs were (a) greater than or equal to 150 , (b) from 75 to 150 , and (c) less than 75 . Also shown is the histogram for (d) the period of AD 1665-1699. The red dashed lines denote 2.0 and 3.0 SDs. The red shaded bars indicate the 24-31 day period.

Tokyo, the signals of the solar rotational period at the times of high solar activity (GSNs $\geq 150$ ) is only 1.5 SDs, while the signal for the middle solar activity (b) is 2.0 SDs. Instead, a period of about 50 days was significant during high solar activity. The signal of solar rotational period was absent at low solar activity (GSNs $<75)$ and during the Maunder Minimum, consistent with the results for Edo and Hachioji. The signal of solar rotational period in Hirosaki and Tokyo in the modern era is in the comparable level as mentioned above; however, the signal in Hirosaki is smaller in the period examined. There might have been an influence from the change in the atmospheric circulation during the time. In our previous study, it was shown that the signal of the solar rotational period was absent on the Japan Sea side in winter when the influence of the Siberian air mass was strong (Miyahara et al., 2017a). In fact, the signal of the solar rotational period in the thunder/lightning activity in Hokkaido in the modern era is only 1.1 SDs (Fig. 2). It is possible that the climate conditions in Hirosaki in the 18th and 19th centuries were closer to those of present Hokkaido. The period of the 18th to 19th centuries corresponds to the late stage of the Little Ice Age. Proxy-based observation of hydroclimate in central Japan during the past 400 years suggested that the Hadley circulation around the western Pacific region had been weakened during the time, lagging behind the de- creases of solar activity and temperatures (Sakashita et al., 2017). Under such condition, it is possible that the influence of the Siberian air mass was stronger or that the seasons under its influence were longer than at present, especially in the northern part of Japan. It implies the possibility that the spatial coverage of the locations under the influence of solar rotation may vary in time depending on the centennial-scale climatological conditions.

Here, we discuss in more detail the solar cycle dependency of the 27-day signals in thunder and lightning activities. As mentioned in the Introduction, possible solar forcing parameters are solar radiation, galactic cosmic rays, and solar wind particles. Interestingly, they have slightly different cyclic behaviors. For example, the 27-day component of the variations in solar radiation and the galactic cosmic rays are enhanced as the number of sunspots is increased. On the other hand, the 27-day component of solar wind parameters is enhanced at the declining phase of solar decadal cycles and sometimes even at the times of low sunspot activity (Owens et al., 2013). If solar wind is the primary source of 27-day variability in meteorological phenomena, we may find such variations to be intensified at the declining phase. We therefore conducted the histogram analysis also for the declining phase of decadal solar cycles. In Fig. S1 in the Supplement, we illustrate the results of the histogram analysis for Hachioji (a), Edo (b), and Hirosaki (c). In this analysis, we defined the periods of declining phase as from +3 years to the +5 years of the maxima of decadal sunspot cycles. The significance levels of the peaks ranging within 24-31 days were $0.7,2.1$, and 0.4 SDs, respectively. The record from Edo exhibits a significant peak; however, it was smaller than that for GSNs $\geq 150$ (Fig. 3d). The signals were not significant in the records from Hachioji and Hirosaki. We also examined the data from the modern period as examined in Miyahara et al. (2017a). In this analysis, we defined the period of declining phase as from AD 1993 to 1995 and from AD 2003 to 2006. We find that the peak is detected with a significance level of about 2.5 SDs; however, it is also less significant compared to the case for solar maxima (Fig. S2d). In the case where we analyze the data for all of the sunspot maxima (from -1 year to +1 year from sunspot cycle maxima) during AD 1700-1868, including maxima GSNs of less than 150, we find peaks around 24-31 days (2.3, 2.3, and 1.5 SDs for Hachioji, Edo, and Hirosaki, respectively; Fig. S2a-c); however, they are also less significant compared to the case for the periods with GSNs $\geq 150$. It confirms that the 27 -day variations in thunder/lightning activities are intensified as the level of sunspot activity increases.

Further examinations are necessary to clarify the origin of the 27-day variations in thunder/lightning activities. Comparisons with solar-related parameters such as solar UV, galactic cosmic rays, and solar wind, which have different characteristics in the 27-day variations and different dependencies on solar cycles, would help in identifying the origin. As a first step, it is needed to trace the propagation of the 27-day 
variations within the climate system based on both modern and premodern data sets. As mentioned in the Introduction, a previous study had demonstrated that the signal of the 27day period in thunder and lightning activity in the modern era indicates a pattern of northeastward migration in Japan. For example, thunder and lightning activities in the area of Kyushu and the adjoining prefectures preceded those of Honshu by approximately 1 day, while there is no time lag among the prefectures in Honshu, except Yamaguchi Prefecture. Although the number of events coincidentally detected in Hirosaki and Edo in the 18th to 19th centuries is very small, the comparison of the two records suggests that it is more likely that there is no time lag between the two areas. Comparisons with the records from further distant locations such as from the Kyushu Island or nearby countries would help in examining the possible migration of the signals.

\section{Conclusions}

Based on the 150-year-long records of thunder and lightning activity retrieved from old diaries kept in Tokyo and Hirosaki in Japan during the 18th to 19th centuries, we examined in detail the persistency and temporal variations in the 27-day solar rotational period in the thunder/lightning activity. We found that the signal of the solar rotational period is most prominent when the sunspot activity is high, that is, when the GSN is greater than or equal to 150 , while the signal is suppressed as the level of sunspot activity decreases. Similar characteristics were confirmed in the records of both Hachioji and Edo in Tokyo. The 27-day component in solar UV and galactic cosmic rays are also enhanced as the level of sunspot activity increases. Our results are consistent with characteristics of such solar forcing parameters; however, further investigations are needed to clarify the detailed mechanisms.

The signal of the solar rotational period in Hirosaki, the northernmost prefecture of the main island, was less significant compared to the signal in Tokyo during the 18th to 19th centuries, contradicting the case in the modern period. It is suggested that the areas that are under the influence of solar rotation might be variable in time depending on the long-term climatological conditions.

Data availability. The weather record extracted from the diaries kept by the Ishikawa family is available at the Historical Weather Database on The Web https://tk2-202-10627.vs.sakura.ne. jp/ (Yoshimura, 2006) as described in Sect. 2 Analysis methods. The weather record in Hirosaki extracted from diaries kept by the Hirosaki Domain have been published by Fukuma $(2010,2014)$. The weather record in Edo extracted from diaries kept by the Hirosaki Domain is to be published by Fukuma.
Competing interests. The authors declare that they have no conflict of interest.

Supplement. The supplement related to this article is available online at: https://doi.org/10.5194/angeo-36-633-2018-supplement.

Acknowledgements. The authors thank Mr. Yoshimi Fukuma for providing the data set of thunder and lightning events in Edo obtained from the diaries kept by the Hirosaki Domain. This work was supported by JSPS KAKENHI grant number 15H05816, 25287051. This work was also supported by the Center for the Promotion of Integrated Sciences of SOKENDAI and the Project to Build an International Collaborative Research for Pre-modern Japanese Texts. We thank Ms. Kana Sakaguchi and Ms. Kyoko Abe for their help in organizing the thunder and lightning data set. We also thank the curators of the Hachioji City Historical Museum for their support in this research.

The topical editor, Marc Salzmann, thanks one anonymous referee for help in evaluating this paper.

\section{References}

Clette, F. and Lefevre, L.: The new sunspot number: assembling all corrections, Sol. Phys., 291, 2629-2651, https://doi.org/10.1007/s11207-016-1014-y, 2016.

Davis, C. J. and Johnson, C. G.: Lightning-induced intensification of the ionospheric sporadic E layer, Nature, 435, 799-801, https://doi.org/10.1038/nature03638, 2005.

Eddy, J. A.: The Maunder Minimum, Science, 192, 1189-1202, 1976.

Füllekrug, M. and Fraser-Smith, A. C.: Further evidence for a global correlation of the Earth-ionosphere cavity resonances, Geophys. Res. Lett., 23, 2773-2776, 1996.

Foukal, P., North, G., and Wigley, T.: A stellar view on solar variations and climate, Science, 306, 68-69, 2004.

Foukal, P., Fröhlich, C., Spruit, H., and Wigley, T. M. L.: Variations in solar luminosity and their effect on the Earth's climate, Nature, 443, 161-166, 2006.

Fukuma, Y.: Browsing the Official Diaries of Hirosaki Clan Government (Preserved by the Hirosaki City Library) Vol. 1, Hopposhinsha, Japan, 2010.

Fukuma, Y.: Browsing the Official Diaries of Hirosaki Clan Government (Preserved by the Hirosaki City Library) Vol. 2, Hopposhinsha, Japan, 2014.

Hong, P. K., Miyahara, H., Yokoyama, Y., Takahashi, Y., and Sato, M.: Implications for the low latitude cloud formations from solar activity and the quasi-biennial oscillation, J. Atmos. Sol.Terr. Phy., 73, 587-591, 2011.

Hood, L. L.: Stratospheric ozone and temperature responses to short-term changes in solar ultraviolet flux: An analysis of Nimbus 7 SBUV and SAMS data, J. Geophy. Res., 91, 5264-5276, 1986.

Hoyt, D. V. and Schatten, K. H.: Group sunspot numbers: A new solar activity reconstruction, Sol. Phys., 181, 491-512, 1998.

Jackman, C. H., Marsh, D. R., Vitt, F. M., Garcia, R. R., Randall, C. E., Fleming, E. L., and Frith, S. M.: Long- 
term middle atmospheric influence of very large solar proton events, J. Geophys. Res., 114, D11304, https://doi.org/10.1029/2008JD011415, 2009.

Kodera, K. and Kuroda, Y.: Dynamical response to the solar cycle, J. Geophys. Res., 107, 4749, https://doi.org/10.1029/2002JD002224, 2002.

Marsh, N. D. and Svensmark, H.: Low cloud properties influenced by cosmic rays, Phys. Rev. Lett., 85, 5004-5007, 2000.

Miyahara, H., Higuchi, C., Terasawa, T., Kataoka, R., Sato, M., and Takahashi, Y.: Solar 27-day rotational period detected in widearea lightning activity in Japan, Ann. Geophys., 35, 583-588, https://doi.org/10.5194/angeo-35-583-2017, 2017a.

Miyahara, H., Aono, Y., and Kataoka, R.: Searching for the 27-day solar rotational cycle in lightning events recorded in old diaries in Kyoto from the 17th to 18th century, Ann. Geophys., 35, 11951200, https://doi.org/10.5194/angeo-35-1195-2017, 2017b.

Muraki, Y., Axford, W. I., Matsubara, Y., Masuda, K., Miyamoto, Y., Menjyou, H., Sakakibara, S., Sako, T., Takami, T., Yamada, T., Shibata, S., Munakata, Y., Munakata, K., Yasue, S., Sakai, T., Mitsui, K., Fujimoto, K., and Flückiger, E.: Effects of atmospheric electric fields on cosmic rays, Phys. Rev. D, 69, 123010, https://doi.org/10.1103/PhysRevD.69.123010, 2004.

Owens, M. J., Challen, R., Methven, J., Henley, E., and Jackson, D. R.: A 27 day persistence model of near-Earth solar wind conditions: A long lead-time forecast and a benchmark for dynamical models, Space Weather, 11, 225-236, https://doi.org/10.1002/swe.20040, 2013.

Owens, M. J., Scott, C. J., Bennett, A. J., Thomas, S. R., Lockwood, M., Harrison, R. G., and Lam, M. M.: Lightning as a spaceweather hazard: UK thunderstorm activity modulated by the passage of the heliospheric current sheet, Geophys. Res. Lett., 42, 9624-9632, 2015.

Sakashita, W., Miyahara, H., Yokoyama, Y., Aze, T., Nakatsuka, T., Hoshino, Y., Ohyama, M., Yonenobu, H., and Takemura, K., Hydroclimate reconstruction in central Japan over the past four centuries from tree-ring cellulose $\delta^{18} \mathrm{O}$, Quatern. Int., 455, 1-7, 2017.
Sato, M. and Fukunishi, H.: New evidence for a link between lightning activity and tropical upper cloud coverage, Geophys. Res. Lett., 32, L12807, https://doi.org/10.1029/2005GL022865, 2005.

Scott, C. J., Harrison, R. G., Owens, M. J., Lockwood, M., and Barnard, L.: Evidence for solar wind modulation of lightning, Environ. Res. Lett., 9, 055004, https://doi.org/10.1088/17489326/9/5/055004, 2014.

Shindell, D., Rind, D., Balachandran, N., Lean, J., and Lonergan, P.: Solar cycle variability, ozone, and climate, Science, 284, 305308, 1999.

Svensmark, H. and Friis-Christensen, E.: Variation of cosmic ray flux and global cloud coverage - A missing link in solar-climate relationships, J. Atmos. Sol.-Terr. Phy., 59, 1225-1232, 1997.

Svensmark, J., Enghoff, M. B., Shaviv, N. J., and Svensmark, H.: The response of clouds and aerosols to cosmic ray decreases, J. Geophys. Res., 121, 8152-8181, 2016.

Takahashi, Y., Okazaki, Y., Sato, M., Miyahara, H., Sakanoi, K., Hong, P. K., and Hoshino, N.: 27-day variation in cloud amount in the Western Pacific warm pool region and relationship to the solar cycle, Atmos. Chem. Phys., 10, 1577-1584, https://doi.org/10.5194/acp-10-1577-2010, 2010.

Tinsley, B. A.: Correlations of atmospheric dynamics with solar wind-induced changes of air-earth current density into cloud tops, J. Geophys. Res., 101, 29701-29714, 1996.

Yamaguchi, Y. T., Yokoyama, Y., Miyahara, H., Sho, K., and Nakatsuka, T.: Synchronized Northern Hemisphere climate change and solar magnetic cycles during the maunder minimum, P. Natl. Acad. Sci. USA, 107, 20697-20702, 2010.

Yoshimura, M.: Historical Weather Database on The Web, available at: https://tk2-202-10627.vs.sakura.ne.jp/, (last access: 10 April 2018), 2006.

Zhou, J. and Tung, K. K.: Observed Tropospheric Temperature Response to 11-year Solar Cycle and What It Reveals About Mechanisms, J. Atmos. Sci., 70, 9-14, 2013. 